\title{
Considering artificial viscosity in a SPH model for simulation of transom waves
}

\author{
Abbas Dashtimanesh, Parviz Ghadimi* \\ Department of Marine Technology, Amirkabir University of Technology, Tehran, Iran \\ *Corresponding author E-mail: pghadimi@aut.ac.ir
}

\begin{abstract}
In the current article, a SPH model based on artificial viscosity is utilized to simulate transom stern flow. In this context, Navier-Stokes equations are discretized in SPH formulation. Furthermore, predictor-corrector algorithm is implemented to solve the discretized equation. Due to density fluctuations, a density filter approach is also included. Finally, a rectangular body is considered as transom and is towed by a constant velocity. Moreover, simulations are performed at two different draft based Froude numbers at which complex physical phenomena have been observed. The obtained results are compared against both experimental and numerical solutions existing in literature. Some capabilities of SPH solution are also illustrated.
\end{abstract}

Keywords: Transom stern; SPH; artificial viscosity; density filter; free surface.

\section{Introduction}

In the last decades, transom sterns have been extensively used for designing stern part of ships. Generally, as design of transom stern has been an important task for engineers, simulation of transom wave has also been an important computational challenge for many researchers. Therefore, experimental studies have been conducted by some of researchers. Doctors [1] was one of first scientists who considered transom stern ventilation. As a results, he developed a formula for transom wave prediction. Doctors [2] also studied previous experimental finding and derived a new regression formula for estimation of transom ventilation. Fu et al [3] performed a series of experiments on R/V Athena model and free surface profile behind the transom stern was measured. Maki et al [4] considered a prismatic model with 20 degrees deadrise angle. They studied ventilation process at transom stern, thoroughly. Two series of experiments were also conducted by Maki et al [5]. They measured transom wave and an empirical relation for transom drying was extended. Fu et al $[6,7]$ tried to present an individual experimental setup for transom flow measurement. They studied transom wave, wave breaking, sinkage and trim of a specific model.

In addition to experimental studies, various numerical models have also been implemented to simulate transom wave, numerically. For the first time, Haussling [8] considered potential solution of transom stern flow. A Rankine Panel method was also utilized by Cheng [9] to study transom stern flow. Boundary element method was used by Scorpio and Beck [10] to examine transom wave. However, their method, similar to Cheng's model [9], gave steady solutions. Therefore, an unsteady finite difference method has been presented by Coleman and Haussling [11] to analyze the unsteady nonlinear waves generated by the stern.

On the other hand, viscous effects were included through the use of the Reynolds-averaged Navier-Stokes equations (RANSE) subject to nonlinear free-surface boundary conditions by Haussling et al. [12]. Tahara and Iwasaki [13] also implemented a RANSE solver to study the transom flow using nonlinear free surface boundary conditions. Furthermore, Schweighofer [14] implemented the RANSE solver FINFLO. However, their simulations, similar to the works of Haussling et al. [12] and Tahara et. Al. [13], were considered to be steady. A steady free surface fitting RANS method was also utilized by Starke et al. [15]. They found that, since the RANS code used was a steady surface fitting method, it did not include all the involved physics such as wave breaking or unsteadiness. Recently, Maki et al. [16] used two different numerical models to study transom stern flow, unsteadily. They implemented a two dimensional level set code as well as commercial software Fluent. The turbulence models were also included. Various nonlinearities in the physical features of the problem were investigated but the obtained free surface profiles were not in satisfactory agreement with experimental results of Maki [17]. For finalization, they stated that "computational tools, although very 
promising, are not yet ready to give a definitive, certain, answer to the problem". However, it has been found that SPH can give reasonable results for problems in which large free surface deformation with high degree of nonlinearity exists. Commonly, smoothed particle hydrodynamics (SPH) is introduced by its Lagrangian nature. Lucy [18] and Gingold and Monaghan [19] were the first who developed SPH. They developed SPH for simulation of astrophysical phenomena. However, the governing equation of astrophysical problems are similar to fluid flow equations. Therefore, Monaghan [20] developed the SPH method for the fluid mechanics problems. Extensive reviews of recent progress and developments in the SPH method are presented by Monaghan [21], Cleary et al [22] and Liu and Liu [23], separately. In the current work, transom stern flow is considered as a complicated fluid flow phenomenon in the marine hydrodynamics. Simulations are performed using smoothed particle hydrodynamics. For more accurate simulations, an artificial viscosity model is also investigated. Furthermore, to remove numerical instabilities in free surface computations, a density filter approach is also implemented. Transom flow, at two draft based Froude numbers is studies and comparisons show excellent capability of the presented SPH model for transom flow simulations.

\section{SPH theory}

In SPH method, computational domain and fluid flow are shown by a set of particles. The movement and the properties of the fluid particles is defined by equations which are expressed as summation interpolants using a kernel function $W$ with smoothing length $h$.

Moreover, SPH is based on integral interpolants. Therefore, any function $A(\mathrm{r})$ can be approximated by:

$A(r)=\int_{\Omega} A\left(r^{\prime}\right) W\left(r-r^{\prime}, h\right) d r^{\prime}$

where $r$ is the vector position. By discretization of approximation 1, the particle approximation of the function at a particle, $a$, can be written as follows:

$A(r)=\sum_{b} m_{b} \frac{A_{b}}{\rho_{b}} W_{a b}$

where all the particles within the region of compact support of the kernel function must be considered at the summation. $W_{a b}=W\left(r_{a}-r_{b}, h\right)$ is the weight function or kernel and $m_{b}$ and $\rho_{b}$ denote the mass and density, respectively.

One of the kernel functions used in this work is the one originally introduced by Monaghan and Lattanzio [24] as in

$W(r, h)=\alpha_{D}\left\{\begin{array}{lr}\frac{3}{4} q^{3}-\frac{3}{2} q^{2}+1 & 0 \leq q \leq 1 \\ \frac{1}{4}(2-q)^{3} & 1 \leq q \leq 2 \\ 0 & q \geq 2\end{array}\right.$

where $\alpha_{D}$ is $10 /\left(7 \pi h^{2}\right)$ in $2 \mathrm{D}$ and $q=r / h . r$ is distance the between two points $a$ and $b$.

\section{Discretization of governing equations in SPH formulation}

The basic governing equations of free surface flows are based on the continuity and momentum Navier-Stokes Equations:

$\frac{d \rho}{d t}=-\rho \nabla \vec{u}$

$\frac{d \vec{u}}{d t}=-\frac{1}{\rho} \nabla \cdot p+v \nabla^{2} \vec{u}+\vec{g}$

where $\rho$ is the density, $p$ is the pressure, $v$ is the kinematic viscosity and the $g$ is the gravity acceleration. Since the fluid is considered to be compressible in the SPH formulation, an equation of state can be used to acquire the fluid pressure. In reality, solving a Poisson's equation is so time consuming but by using an equation of state, the determination of fluid pressure, in the case of compressible fluids, can be facilitated. In present simulations, Tait's equation of state is implemented: 


$$
P=B\left[\left(\frac{\rho}{\rho_{0}}\right)^{\gamma}-1\right]
$$

where $\rho_{0}=1000\left(K g=m^{3}\right), \gamma$ is the polytrophic constant, usually between 1 and 7 . The parameter B is also a constant related to the bulk modulus of elasticity of the fluid. For imposing the zero pressure at a surface, the minus one term in the equation of state is utilized. In the meantime, the continuity equation, in SPH form, can be written as follows:

$\frac{d \rho_{a}}{d t}=\sum_{b} m_{b} v_{a b} \nabla_{a} W_{a b}$

Furthermore, in this study, the artificial viscosity introduced by Monaghan [25] is considered to describe the momentum equation in the SPH method. The artificial viscosity is picked due to its simplicity. In SPH formulation, Eq.5 can be written as

$\frac{d v_{a}}{d t}=-\sum_{b} m_{b}\left(\frac{P_{a}}{\rho_{a}^{2}}+\frac{P_{b}}{\rho_{b}^{2}}+\Pi_{a b}\right) \nabla_{a} W_{a b}+g$.

The pressure gradient term in symmetrical form is expressed in SPH notation as

$-\frac{1}{\rho} \nabla P=-\sum_{b} m_{b}\left(\frac{P_{a}}{\rho_{a}^{2}}+\frac{P_{b}}{\rho_{b}^{2}}\right) \nabla_{a} W_{a b}$

where $P$ and $\rho$ are pressure and density. Also, $\Pi_{a b}$ is the viscosity term given as

$\Pi_{a b}= \begin{cases}\frac{-\alpha \overline{c_{a b}} \mu_{a b}}{\overline{\rho_{a b}}} & \text { if } v_{a b} . r_{a b}<0, \\ 0 & \text { otherwise }\end{cases}$

with

$\mu_{a b}=\frac{h v_{a b} \cdot r_{a b}}{r_{a b}^{2}+\eta^{2}}$

where $\overline{\rho_{a b}}=\frac{1}{2}\left(\rho_{a}+\rho_{b}\right), \overline{c_{a b}}=\frac{1}{2}\left(c_{a}+c_{b}\right), \eta^{2}=0.01 h^{2}$ and $\alpha$ is a free parameter that can be changed according to each problem.

\section{Numerical details}

In this section, some numerical details implemented in our SPH model are introduced.

\subsection{Moving the particles}

XSPH variant is used to move the Particles in time stepping procedure. The velocity of a particle $a$ will be modified by this technique. This velocity is recalculated taking into account the velocity of that particle and the average velocity of the closest neighboring particles that are interacting with particle $a$.

$\frac{d r_{a}}{d t}=v_{a}+\varepsilon \sum_{b} \frac{m_{b}}{\bar{\rho}_{a b}} v_{b a} W_{a b}$

where $\rho_{a b}=\left(\rho_{a}+\rho_{b}\right) / 2$ and $\varepsilon$ is a constant.

\subsection{Time stepping procedure}

This scheme predicts the evolution in time as

$v_{i}^{n+1 / 2}=v_{i}^{n}+\frac{\Delta t}{2} F_{i}^{n} ; \rho_{i}^{n+1 / 2}=\rho_{i}^{n}+\frac{\Delta t}{2} D_{i}^{n} ; r_{i}^{n+1 / 2}=r_{i}^{n}+\frac{\Delta t}{2} V_{i}^{n}$

calculating $p_{i}^{n+1 / 2}=f\left(\rho_{i}^{n+1 / 2}\right)$ according to the equation of state mentioned above. These values are then modified using forces at the half step

$v_{i}^{n+1 / 2}=v_{i}^{n}+\frac{\Delta t}{2} F_{i}^{n+1 / 2} ; \quad \rho_{i}^{n+1 / 2}=\rho_{i}^{n}+\frac{\Delta t}{2} D_{i}^{n+1 / 2} ; r_{i}^{n+1 / 2}=r_{i}^{n}+\frac{\Delta t}{2} V_{i}^{n+1 / 2}$

Finally, at the end of any time step, the velocity values are calculated as follows: 
$v_{i}^{n+1}=2 v_{i}^{n+1 / 2}-v_{i}^{n} ; \quad \rho_{i}^{n+1}=2 \rho_{i}^{n+1 / 2}-\rho_{i}^{n} ; r_{i}^{n+1}=2 r_{i}^{n+1 / 2}-r_{i}^{n}$

In addition, the pressure is calculated from the density using $p_{i}^{n+1}=f\left(\rho_{i}^{n+1}\right)$ relation.

\subsection{Density filter}

The density of any particle may be easily evaluated by implementing the following equation:

$\rho_{i}=\sum_{j=1}^{N} m_{j} W_{i j}$.

Meanwhile, this relation leads to numerical instabilities in free surface computations. In order to make the computation stable, several modifications have been implemented in the present work. A moving least squares (MLS) approach which was proposed by Colagrossi and Landrini [26] is applied in the developed code. It is a first order correction and as such, the linear variation of the density field can be exactly reproduced:

$\bar{\rho}_{a}=\sum_{b} \rho_{b} W_{a b}^{M L S} \frac{m_{b}}{\rho_{b}}=\sum_{b} m_{b} W_{a b}^{M L S}$

The corrected kernel is also evaluated as follows:

$W_{a b}^{M L S}=W_{a b}^{M L S}\left(r_{a}\right)=\beta\left(r_{a}\right) \cdot\left(r_{a}-r_{b}\right) W_{a b}$

The kernel can exactly reproduce linear variations in the density field, if used at every time step. Therefore, this modification is used to reinitialize the density field at 300 time steps.

\subsection{Boundary Conditions}

In this work, dynamic boundary condition [27] is applied. Boundary particles have a similar behavior to fluid particles and follow continuity, momentum and state equations. Contrary to the fluid particles which have displacement, boundary particles' displacements are zero. Arrangement of boundary particles is displayed in Fig.1.

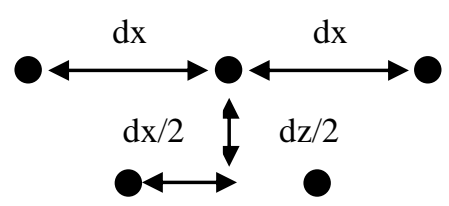

Fig.1: Interaction of fluid particles and boundary particles.

When a particle reaches the boundaries, density of the boundary particles is increased. Because of the pressure term P/ $\rho$ in the momentum equation, the force which is exerted on the fluid particle increases. When the distance between the boundary particle and fluid particle decreases, the density, pressure and acting force on the fluid particle increase by a repulsive mechanism.

\section{Results and discussions}

To study the transom stern flow, a combination of artificial viscosity, predictor-corrector algorithm, XSPH variant and MLS density filter are utilized. Based on our knowledge from literature, some free-surface fluctuations can be observed at $\mathrm{Fn}_{\mathrm{T}}=2$ (draft based Froude number). Furthermore, the $\mathrm{Fn}_{\mathrm{T}}=2.5$ is characterized by a breaking wave on the first crest behind the body. Due to computational restrictions, transom flow is only simulated at two mentioned Froude numbers. Generally, most of the nonlinear features of transom stern flow are included in these Froude numbers.

Moreover, transom is considered as a rectangular body and towed by a constant velocity. Transom draft is also supposed to be $\mathrm{T}_{\mathrm{d}}$. Generally, computational domain is depicted in Fig.2 and it assumed to be extended enough (horizontally and vertically). 


\section{Body}

Free Surface

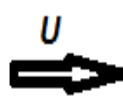

\section{Computational Domain}

Fig.2: Computational domain

The obtained results are compared against both experimental [17] and unsteady numerical solutions of [16]. Afterward, the nonlinear features of problem, the free surface fluctuations and the generated breaking wave behind the transom are illustrated.

The root mean squared (RMS) of free surface profile is presented at the considered Froude numbers. It is observed that SPH solutions offer reasonable results, relatively. For validation of the current studies, maximum RMS of free surface profile, the dimensionless amplitude of first $\left(\mathrm{a}_{1}\right)$ and second $\left(\mathrm{a}_{2}\right)$ wave crest and their slopes will be compared against other numerical results such as Fluent and level set computations to answer this question of whether the SPH could accurately simulate the transom stern flow over previous numerical solutions.

Figure 3 presents a comparison among the current numerical finding, the experimental and Fluent solutions as well as level set results. Although, both Fluent and SPH give same accuracy at $\mathrm{Fn}_{\mathrm{T}}=2$, but it is fairly clear that SPH can be more favorable due to its accuracy at both Froude numbers. To prove this declaration, Figs. 4 and 5 compare the SPH's wave characteristics with the corresponding results of [16] and [17]. Therefore, it is proved that, at the considered Froude numbers, both RMS and wave characteristic of SPH solution is in better agreement with the experimental data in comparison with numerical solution of Maki et al. [16].

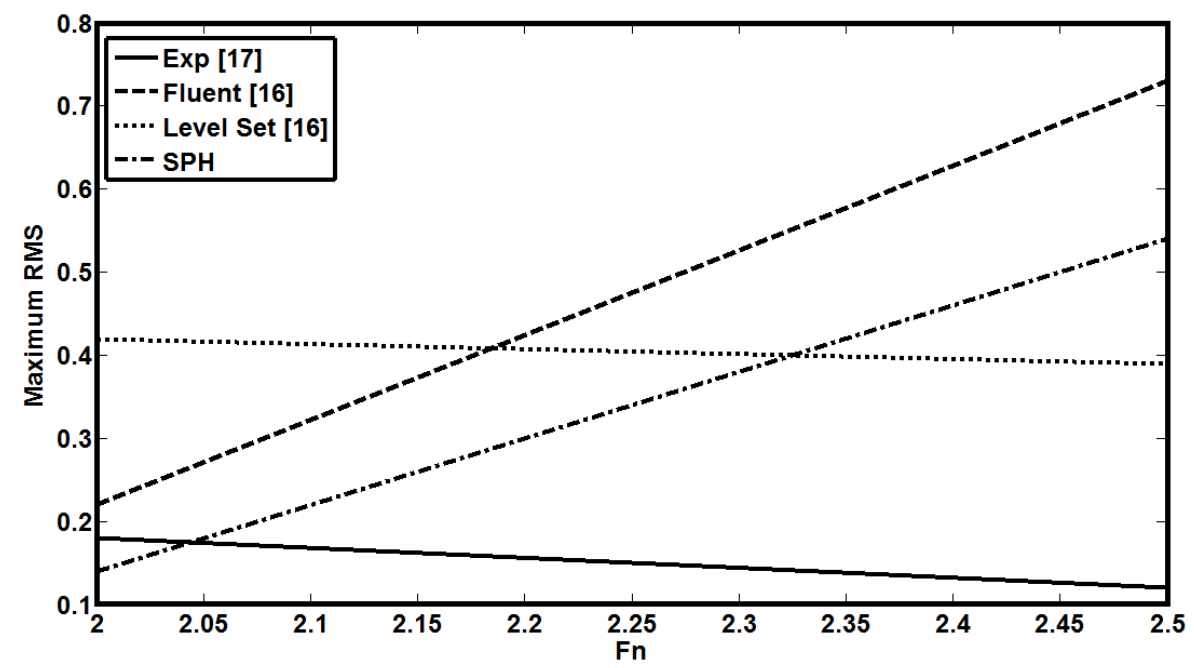

Fig.3: Maximum RMS of the obtained free surface profiles at $\mathrm{Fn}_{\mathrm{T}}=2$ and $\mathrm{Fn}_{\mathrm{T}}=2.5$ 


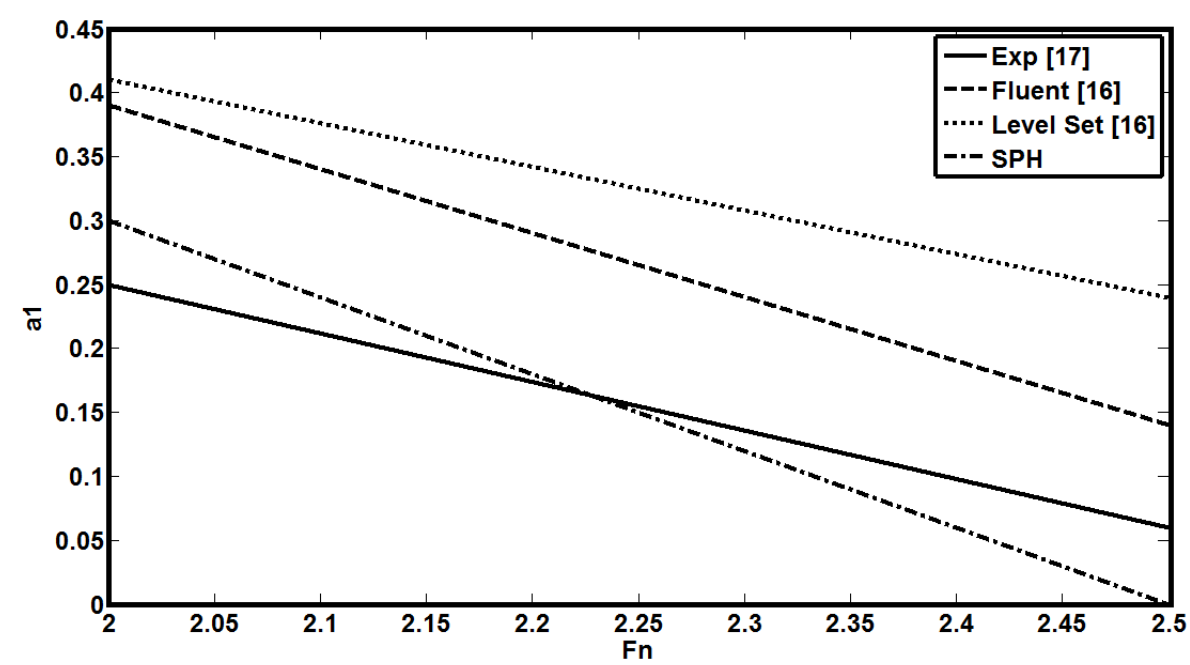

Fig.4: Dimensionless amplitude of first wave crest at $F n_{T}=2$ and $F n_{T}=2.5$

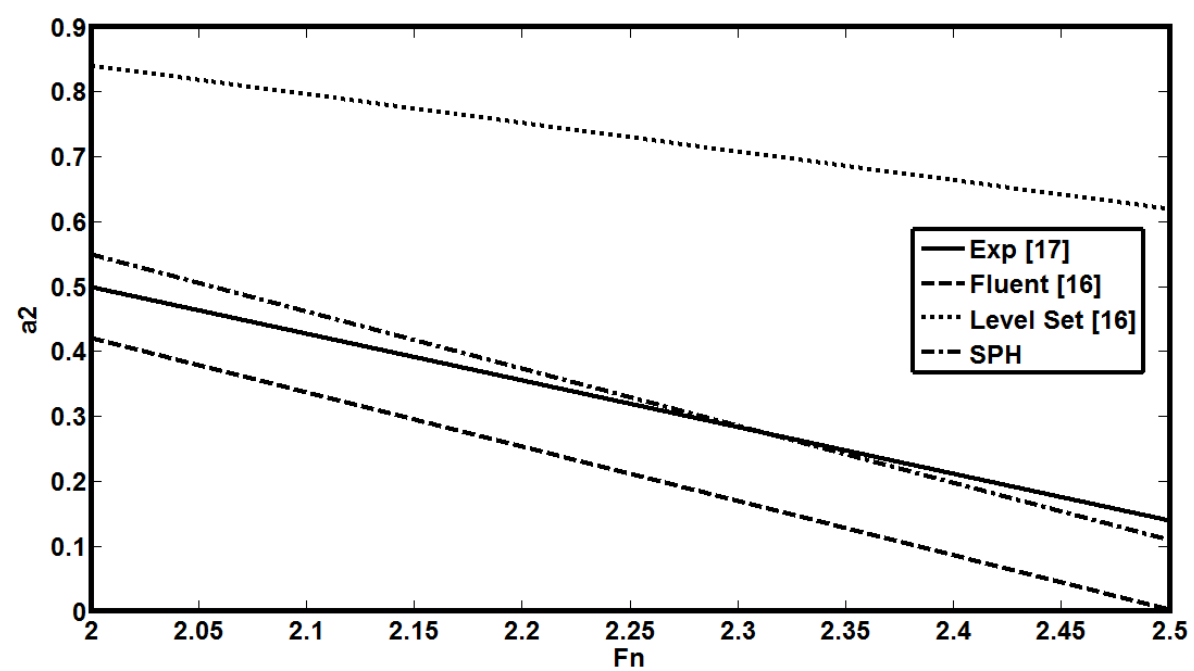

Fig.5: Dimensionless amplitude of second wave crest at $F n_{T}=2$ and $F n_{T}=2.5$

Furthermore, for further justification of the new results, wave slopes of first and second wave crest considered above are also presented in Figs.6 and 7. From these illustration, it can finally be concluded that SPH simulation of transom stern flow is a step forward in the challenging problem of computing flows about transom sterns.

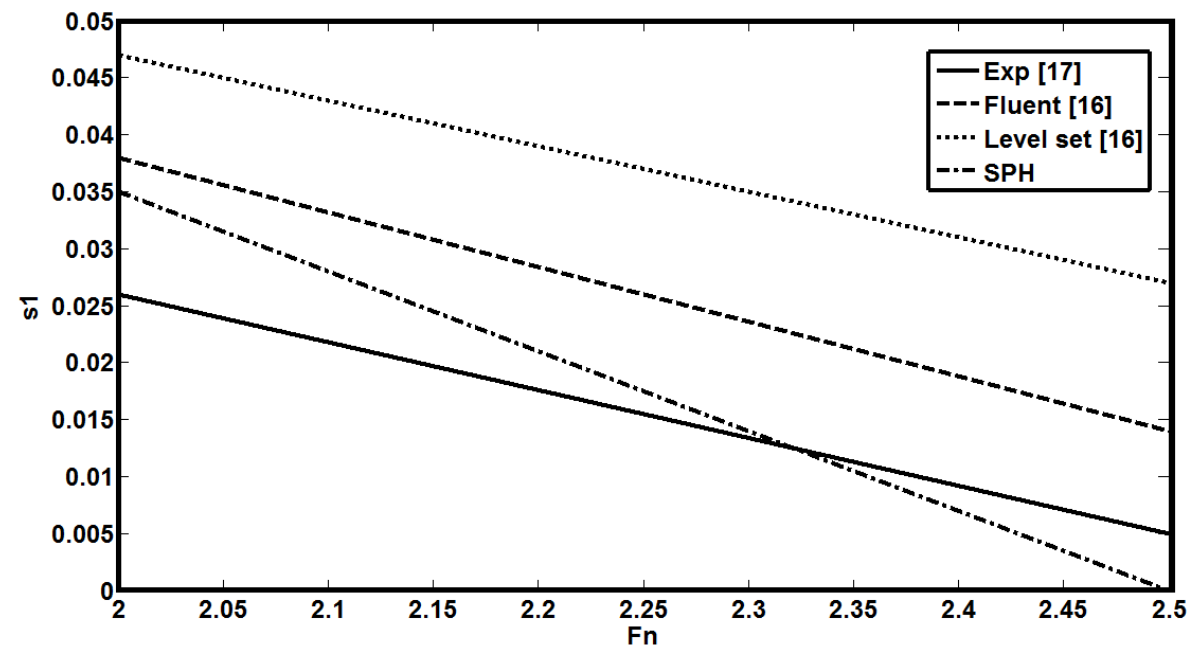

Fig.6: Non-dimensional wave slope of first wave crest at $\mathrm{Fn}_{\mathrm{T}}=2$ and $\mathrm{Fn}_{\mathrm{T}}=2.5$ 


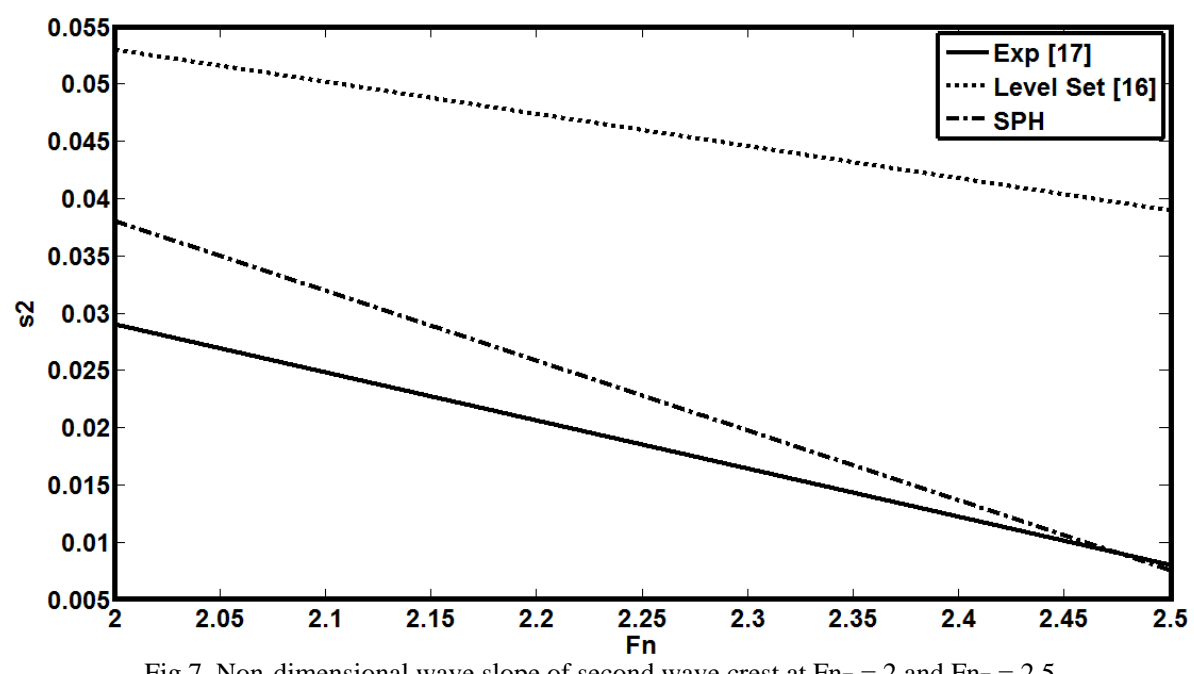

Fig.7. Non-dimensional wave slope of second wave crest at $\mathrm{Fn}_{\mathrm{T}}=2$ and $\mathrm{Fn}_{\mathrm{T}}=2.5$

\section{Nonlinear features of transom stern wave}

In this section, some physical nonlinearities of the problem which are simulated using SPH are illustrated. Figure 8 shows large free surface fluctuation which occurs at lower Froude number. It is very clear that transom is partially ventilated and generally some vortex shedding is observed in free surface just behind the transom region. Nature of vortex shedding in transom region must be considered in future studies, separately. After increasing the Froude number, transom will be ventilated at a certain Froude number and some wave breaking also happens. Figure 9 show has simulated wave breaking using SPH just behind the transom stern. Physics of the wave breaking is a complicated problem and will be considered in next articles.

It is fairly clear that SPH could simulate air entrainment into water as well as droplet spray. Simulation of these complex hydrodynamic phenomena is very important in many applications. However, it must be mentioned that precise study of water spray and air entrainment need millions of particles while only 480,000 particles was considered her, due to computational limitations.

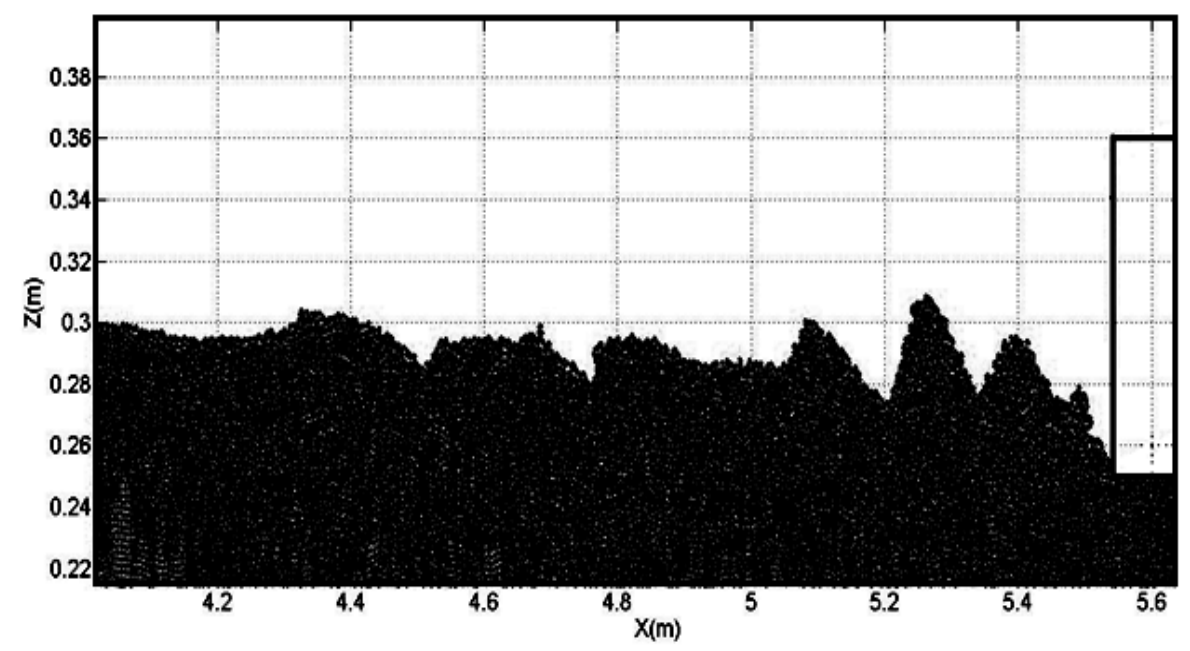

Fig.8: Large free surface fluctuations 


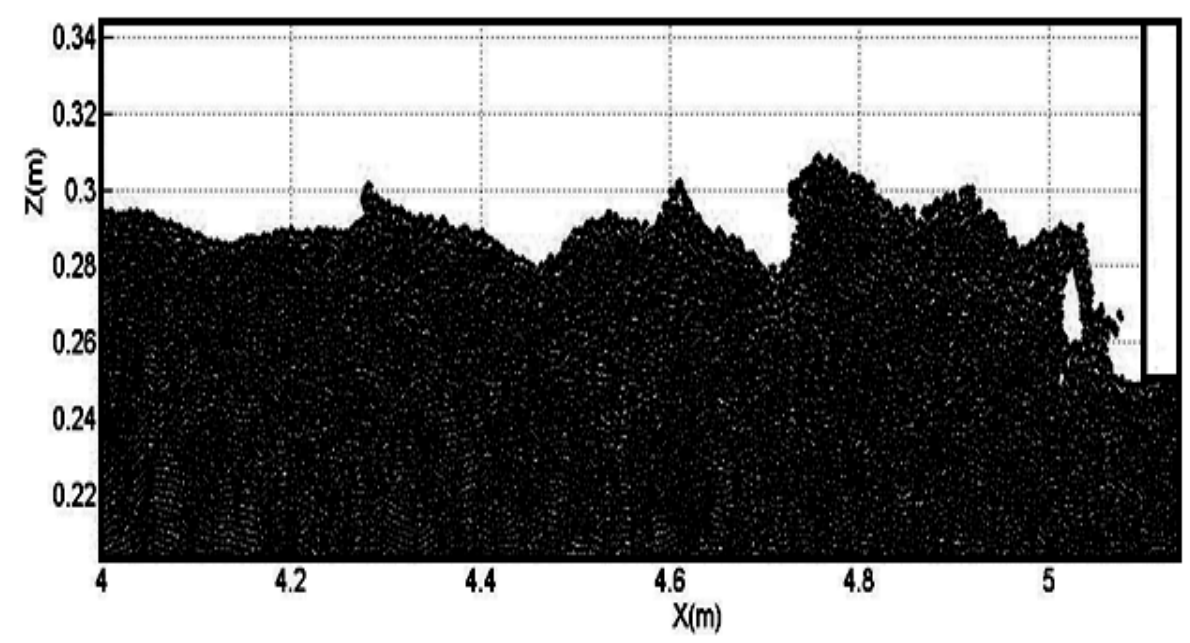

Fig.9: Breaking Wave occurrence

\section{Conclusions}

In the current article, a SPH model based on artificial viscosity is developed for simulation of transom wave. To save computational time, an equation of state instead of Poisson's equation of pressure is utilized. For time steeping calculation, XSPH variant in conjunction with predictor-corrector scheme are used. Moreover, MLS density filter is implemented to remove density fluctuations which affect the pressure and free surface profile. Computations are considered at two different draft based Froude numbers. Transom stern is also considered as a rectangular body and towed by a constant velocity at desirable transom draft. The obtained results are compared against both experimental and numerical data. Comparisons show that SPH is a step forward in numerical simulation of transom stern flow. Furthermore, large free surface fluctuations as well as wave breaking, which are two examples for nonlinearity of transom waves, are illustrated. Consideration of a wide range of Froude numbers as well as study of vortex shedding behind the transom can be considered as future studies.

\section{References}

[1] Doctors L, Macfarlane G, Young R, A study on Transom Stern Ventilation, International Shipbuilding Progress, 2007.

[2] Doctors J, Hydrodynamic of the Flow Behind a Transom Stern, Proceeding of the 29th Israel Conference on Mechanical Engineering, Haifa, 2003.

[3] Fu T, Karion A, Pence A, Rice J, Walker D, Ratcliffe T, Characterization of the Steady Wave Field of the High Speed Transom Stern Ship Model 5365 Hull Form, Carderock Division, Naval Surface Warfare Center, Hydromechanics Department Report, 2005.

[4] Maki J.K., Troesch A, Beck R, Qualitative Investigation of Transom Stern Flow Ventilation, 2005.

[5] Maki J.K, Doctors J.L, Beck R, Troesch A, Transom-Stern Flow for High-Speed Craft, 8th International Conference on Fast Sea Transportation, Saint Petersburg, Russia, 2005.

[6] Fu T, Fullerton A, Ratcliffe T, Minnick L, Walker D, Lee Pence M, Anderson K, A Detailed Study of Transom Breaking Waves, Carderock Division, Naval Surface Warfare Center, Hydromechanics Department Report, 2009.

[7] Fu T, Fullerton A, Drazen D, Minnick L, Walker D, Ratcliffe T, Russell L, Capitain M, A Detailed Study of Transom Breaking Waves: Part II, Carderock Division, Naval Surface Warfare Center, Hydromechanics Department Report, 2010.

[8] Haussling, H., "Two Dimensional Linear and nonlinear Stern Waves”, J. Fluid Mech, 1980, Vol. 97, pp: 759-769.

[9] Cheng, B., "Computations of 3D Transom Stern Flows", Proc. Fifth International Conference on Numerical Ship Hydrodynamics, National Academy Press: Washington, DC, 1989, pp: 581-592.

[10] Scorpio, S. and Beck, R.F., "Two-Dimensional Inviscid Transom Stern Flow”, Proceedings 12th International Workshop on Water Waves and Floating Bodies, Carry-le-Rouet, France, 1997, pp: 221-226.

[11] Coleman, R.M., Haussling, H.J., "Nonlinear Waves Behind an Accelerated Transom Stern", Proc. of the Third Int. Conf. on Numerical ship Hydrodynamics, Paris, 1981, 111.

[12] Haussling, H.J., Miller, R.W., Coleman, R.M., "Computation of High Speed Turbulent Flow about a Ship Model with a Transom Stern", Carderock Division, Naval Surface Warfare Center, Hydromechanics Department Report, 1997.

[13] Tahara, Y., Iwasaki, Y., “A Study of Transom Stern Free Surface Flow by 2D Computational and Experimental Models”, J. Kansai Soc, N. A., Japan, 1997, Vol. 227.

[14] Schweighofer, J., "Investigation of Two-Dimensional Transom Waves Using Inviscid and Viscous Free-Surface Boundary Conditions at Model- and Full-Scale Ship Reynolds Numbers”, 5th Numerical Towing Tank Symposium, Pornichet, France, 2005.

[15] Starke, B., Ravan, H., Van Der Ploeg, A., "Computation of transom-stern flows using a steady free-surface fitting RANS method", 9th International Conference on Numerical Ship Hydrodynamics, Michigan, 2007. 
[16] Maki, K., Iafrati, A., Rhee, S., Beck, R., Troesch, A., “The Transom-Stern Modeled as a Backward Facing Step, 26th Symposium on Naval Hydrodynamics, Rome, Italy, (2006).

[17] Maki. K.J., "Transom Stern Hydrodynamics”, Doctoral Thesis, University of Michigan, 2006.

[18] Lucy L, A numerical approach to the fission hypothesis, Astron J., pp: 82-1013, 1977.

[19] Gingold R.A., Monaghan J. J., Smoothed particle hydrodynamics: theory and application to non-spherical stars, Mon Not Roy Astron Soc, pp: $181-375,1977$.

[20] Monaghan J. J., Simulating free surface flows with SPH, J. Comput. Phys, Vol. 110, pp: 399-406, 1994.

[21] Monaghan J.J., Smoothed particle hydrodynamics, Reports on Progress in Physics, Vol. 68, pp: 1703-1759, 2005.

[22] Cleary P.W., Prakash M., Ha J., Stokes N., Scott C, Smooth Particle hydrodynamics: status and future potential, Progress in Computational Fluid Dynamics Vol. 7, pp: 25-76, 2007.

[23] Liu M.B., Liu G.R., "Smoothed Particle Hydrodynamics (SPH): an Overview and Recent Developments, Archives of Computational Methods in Engineering, Vol. 17, Issue 1, pp: 25-76, 2010.

[24] Monaghan, J. J. and Lattanzio, J. C., “A refined method for astrophysical problems”, Astron. Astrophys, Vol. 149, pp: 135-143, 1985.

[25] Monaghan, J. J., "Smoothed Particle Hydrodynamics", Annual Rev. Astron. Appl., Vol. 30, pp: 543-574, 1992.

[26] Colagrossi, A., Landrini, M., "Numerical Simulation of Interfacial Flows by Smoothed Particle Hydrodynamics", J. Comp. Phys., 2003, Vol. 191, pp. 448-475.

[27] Liu, GR., Liu, MB., "Smoothed particle hydrodynamics: a mesh free particle method”, World Scientific, Singapore, 2003. 